\title{
Trivium
}

Revue franco-allemande de sciences humaines et sociales - Deutsch-französische Zeitschrift für Geistesund Sozialwissenschaften

$3 \mid 2009$

Droits subjectifs et droits de l'homme

\section{Subjektive Rechte und Menschenwürde. Zur Einleitung}

Christoph Menke

\section{OpenEdition}

Journals

Édition électronique

URL : http://journals.openedition.org/trivium/3296

DOI : 10.4000/trivium.3296

ISSN : 1963-1820

Éditeur

Les éditions de la Maison des sciences de l'Homme

\section{Référence électronique}

Christoph Menke, «Subjektive Rechte und Menschenwürde. Zur Einleitung », Trivium [En ligne], 3|

2009, mis en ligne le 15 avril 2009, consulté le 22 septembre 2020. URL : http://

journals.openedition.org/trivium/3296 ; DOI : https://doi.org/10.4000/trivium.3296

Ce document a été généré automatiquement le 22 septembre 2020.

\section{(c) (i) $(9)$}

Les contenus des la revue Trivium sont mis à disposition selon les termes de la Licence Creative Commons Attribution - Pas d'Utilisation Commerciale - Pas de Modification 4.0 International. 


\title{
Subjektive Rechte und Menschenwürde. Zur Einleitung
}

\author{
Christoph Menke
}

\section{Zwei Lesarten subjektiver Rechte}

1 In seinen Untersuchungen zum Recht moderner Gesellschaften hat Max Weber die Figur subjektiver Rechte als das Ergebnis eines grundlegenden Wandels im Verständnis von "Berechtigung" gedeutet. Das traditionelle Recht erlegt dem Einzelnen Verpflichtungen auf, sagt ihm, was er tun soll und bedroht ihn mit den Folgen, die eine Unterlassung des Zutuenden haben. Eine solche Ordnung der Verpflichtungen schafft auf ihrer Rückseite - »in der Form eines $>$ Reflexes « ${ }^{1}{ }^{1}$ - Berechtigungen. Das gilt in dem elementaren Sinn, dass die rechtliche Auferlegung und Durchsetzung der Verpflichtung, zum Beispiel nicht zu stehlen, auf der anderen Seite die Berechtigung hervorbringt, nicht bestohlen zu werden. Aber sie gibt dem anderen kein »subjektives Recht» darauf, nicht bestohlen zu werden. Von einem Recht auf Eigentum (oder gar Leben, Freiheit usw.) spricht erst das moderne Recht - und dreht damit die Perspektive um: Die Berechtigung des einen ist nicht mehr »Reflex« der Verpflichtung der anderen, sondern bringt diese Verpflichtung hervor. Die Berechtigung des Einzelnen wird zum Können, zur »Machtquelle« (Weber). Das ist die Berechtigung im Sinn sogenannter »subjektiver Rechte«: Subjektive Rechte ermächtigen; sie geben dem Einzelnen Macht über das Tun der Anderen.

2 In seiner Untersuchung des modernen Rechts hat Weber auch erklärt, weshalb es zur Einführung dieser neuen Figur des subjektiven Rechts kam. Weber nennt zwei verschiedene Faktoren, deren spannungsvolles Nebeneinander hinter den Gegensätzen steht, die bis heute die Diskussionen um die subjektiven Rechte prägen: „Die entscheidende Triebkraft für diese Veränderung waren: politisch das Machtbedürfnis der Herrscher und Beamten der erstarkenden politischen Staatsgewalt, ökonomisch aber [...] die Interessen der Marktmachtinteressenten, d.h. also: der durch Besitz als solchen (`Klassenlage`) im formal `freien` Preis- und Konkurrenzkampf auf dem Markt 
ökonomisch Privilegierten. $\aleph^{2}$ Es sind also zwei Faktoren, die die neue Form subjektiver Rechte hervortreiben. Der eine Faktor ist die grundlegende Veränderung der politischen Ordnung: Die Vielzahl intermediärer Ordnungen der feudalen Gesellschaften werden durchschlagen durch die Entstehung des modernen Staates, der die Gewalt monopolisiert und dadurch unmittelbar allen seinen Bürgern gegenübersteht. Subjektive Rechte beziehen sich auf dieses Verhältnis zwischen Bürger und Staat, das die moderne politische Ordnung definiert. Der andere Faktor ist die Entstehung einer gesellschaftlichen Sphäre, die von normativ regulierenden politischen Steuerungen freigesetzt ist. Dadurch wird das Recht zur Schutz- und Sicherungsinstanz all derjenigen Vereinbarungen, die die Mitglieder der bürgerlichen Gesellschaft "formal 'frei«" (Weber, mit Marx) getroffen haben. Subjektive Rechte geben ihnen »Kompetenzen ${ }^{3}$, die Sicherheit, dass ihre Verträge rechtlich geschützt sind.

Durch diese doppelte Funktion für moderne Gesellschaften zieht eine Zweideutigkeit in die Figur subjektiver Rechte ein, die bis in die gegenwärtigen Debatten hinein bestimmend geblieben ist. Das zeigt sich exemplarisch an der unaufgelösten Spannung im Verständnis rechtlich verbürgter Freiheit. Schon bei Kant stoßen im Begriff des Rechts die Idee vernünftiger Autonomie und der Begriff privater Willkür hart aufeinander. ${ }^{4}$ Diese Spannung spiegelt sich in zwei ganz verschiedenen Deutungen der Freiheit, die durch subjektive Rechte verbürgt wird:

4 Auf der einen Seite steht die exemplarisch von Amartya Sen vertretene These, dass der Sinn der Ermächtigung durch subjektive Rechte in der »Befähigung« von Subjekten zur Teilnahme an sozialen Praktiken besteht. ${ }^{5}$ Rechtlich verbürgte Freiheit ist Freiheit als subjektive Fähigkeit, und subjektive Fähigkeiten sind die zur Ausübung sozialer Praktiken, zur sozialen Teilnahme. Sen gibt eine soziale Reformulierung des Begriffs der Autonomie und sieht in ihrer Ermöglichung den Sinn der Rechte. Damit zieht Sen die Konsequenz aus dem Bruch mit der traditionell liberalen Idee des Schutzes »negativer Freiheit $\aleph^{6}$, den der gegenwärtige US-amerikanische Liberalismus - Ronald Dworkin, John Rawls, in anderer Form: Joseph Raz - vorgenommen hat: Wenn die Idee subjektiver Rechte von den falschen Ideologien des Subjekts befreit wird, ist es nichts anderes als ein Medium der sozialen Befähigung zur Autonomie.

5 Auf der anderen Seite steht die These, dass es zur Form subjektiver Rechte gehört, dass sie Rechtstitel ausstellen, deren Inanspruchnahme in die Willkür des Einzelnen gestellt ist. Subjektive Rechte zu haben bedeutet nur die Möglichkeit ihrer Inanspruchnahme; die entsprechende Verpflichtung der anderen ist insoweit in das Belieben des Rechtsinhabers gestellt. ${ }^{7}$ In seiner Analyse der Figur des subjektiven Rechts hat Niklas Luhmann diesen entscheidenden Punkt so formuliert: »Es [sc. das Recht] will nur wollen, wenn und soweit der Einzelne will und nicht wollen, wenn der Einzelne nicht will; es nimmt seinen Willen nach Maßgabe privaten Wollens zurück. ${ }^{8}$ Die Freiheit, die subjektive Rechte eröffnen, ist demnach hier nicht als die Fähigkeit zur sozialen Teilnahme, sondern als die Eröffnung von Spielräumen der privaten Willkür verstanden. ${ }^{9}$ Die Form der subjektiven Rechte, gleichgültig was sie beinhalten, bedeutet die Ermächtigung des Einzelnen in seinem normativ freigesetzten Willkür-Willen.

\section{Subjektive Rechte und Menschenwürde}

6 Es finden sich mithin zwei spannungsvoll entgegengesetzte Bestimmungen des Subjekts der Rechte: als soziales Mitglied und als privates Individuum. Dieser Gegensatz 
in der Deutung der Form subjektiver Rechte wiederholt sich darin, wie das Verhältnis zwischen dieser Form und dem normativen Gehalt der Menschenrechte bestimmt wird. Dabei kann man zunächst sagen, dass die Menschenrechte eine bestimmte Klasse von subjektiven Rechten sind; nämlich die grundlegenden: diejenigen Rechte, in denen es um den Status des Rechtssubjekts als solchen geht. Diese werden in der Regel als drei Arten von Rechten verstanden: die liberalen Abwehrrechte, die demokratischen Teilnahmerechte und die sozialen Leistungsrechte. Eine erste Frage richtet sich dann darauf, ob bereits die bloße Form subjektiver Rechte diese anspruchsvollen normativen Gehalte in sich trägt. Wird diese Frage bejaht, dann kann man argumentieren, dass es ein Widerspruch ist, wenn Staaten, wie etwa asiatische Entwicklungsdiktaturen, eine kapitalistische Eigentumsordnung ohne grundlegende Rechte einführen. ${ }^{10}$

Die Frage nach den Menschenrechten ist aber nicht nur die Frage nach dem Gehalt grundlegender subjektiver Rechte, sondern nach ihren Trägern; nicht nur die Frage also, welche Rechte gelten (sollen), sondern wer solche grundlegenden Rechte hat oder haben soll. Diese Frage wird in der deutschen Diskussion zumeist unter dem Titel "Menschenwürde« behandelt. Den Bezugspunkt bildet dabei Artikel 1 des deutschen Grundgesetzes von 1949: „Die Würde des Menschen ist unantastbar. Sie zu achten und zu schützen ist Verpflichtung aller staatlichen Gewalt.« Für das Grundgesetz gilt dabei derselbe Zusammenhang zwischen der Berufung auf die Menschenwürde und der Erfahrung der totalitären "Akte der Barbarei«, den die Allgemeine Erklärung der Menschenrechte von 1948 in ihrer Präambel ausdrücklich herstellt. ${ }^{11}$ Der Begriff der Menschenwürde sagt mithin: Jeder Mensch ist der Anerkennung als ein Träger subjektiver Rechte würdig.

8 In der deutschen Philosophie und Rechtswissenschaft war lange Zeit die Ansicht vorherrschend, der Begriff der Menschenwürde bezeichne einen obersten normativen »Wert«, der der politischen Ordnung unverfügbar vorgegeben sei; diese Ansicht konnte dann weiterhin entweder thomistisch oder kantisch formuliert werden..$^{12}$ Stets wird dabei eine vor- oder gar überpolitische Werteordnung postuliert, die sich in moralischer Erkenntnis erfassen läßt und der gegenüber die Politik in die bloße Rolle einer »Anwendung" gerät. Diese Position ist ebenso aus rationalitäts- wie politiktheoretischen Gründen zunehmend in die Kritik geraten (auch wenn sie in den jüngeren bioethischen Debatten wieder eine Rolle spielt) ${ }^{13}$. Dass die "Menschenwürde« keinen unabhängigen Ableitungsgrund für die Rechtsordnung bilden kann, bedeutet aber nicht, dass der Begriff seine Bedeutung für die Bestimmung der Rechte verloren habe. Diese Bedeutung liegt in dem Beitrag, den der Begriff der Menschenwürde zur Klärung der oben formulierten Frage leistet: wer der Träger subjektiver Rechte ist und damit, wie das Subjekt solcher Rechte verstanden werden muss.

Der Begriff der Würde beantwortet die Frage nach dem Subjekt der Rechte, indem er als dieses Subjekt den "Menschen" bezeichnet. Dabei liegt die Emphase dieser Antwort darauf, dass alle (Menschen) Träger von Rechten sind; dass also niemand von der Rechtssubjektivität auszuschließen ist. Aber die Emphase dieser Antwort täuscht darüber hinweg, dass der Bezug auf den »Menschen« als Subjekt der Rechte eine spezifische Adressierung impliziert. Das zeigt sich am deutlichsten an den Fallstricken des Naturalismus, die den Begriff des »Menschen« umgeben. So hat bereits Hegel das Argument formuliert, dass sich die Rechtsfähigkeit nicht auf »den Menschen als Naturwesen « beziehen kann; denn es sind gerade der natürliche Mensch und seine Unterschiede an Fähigkeiten, auf die sich die Rechtfertigung der Sklaverei bezieht, 
gegen die die These, dass alle Menschen Rechtssubjekte sind, gerichtet ist. ${ }^{14}$ Diese Kritik der Naturalisierung, die im Begriff des Menschen liegt, hat Hannah Arendt ${ }^{15}$ nachdrücklich reformuliert und dabei zugleich darauf hingewiesen, wie ein nichtnaturalistisches Verständnis die Würde des Menschen zu verstehen hätte: Sie muss den Menschen nicht als natürliches, sondern als essentiell "politisches« (Arendt) oder "geistiges" (Hegel) Wesen nehmen. Würde und damit Rechtsfähigkeit hat der Mensch, sofern es seine Bestimmung ist, soziales Mitglied zu sein. Das ist sein »Recht auf Rechte.$^{16}$

Diese Antwort, die der Begriff der Menschenwürde auf die Frage nach dem Subjekt der Rechte gibt, entspricht der ersten der beiden oben genannten Bestimmungen subjektiver Rechte: der Rechte als Ermöglichung sozialer Teilnahme. Es macht jedoch die Dialektik der »Menschenwürde« aus, dass der Begriff des Menschen zugleich den Grund für die entgegengesetzte, individualistische Lesart subjektiver Rechte bildet. Der Begriff der Menschenwürde will in dieser zweiten Lesart sagen, dass der Mensch in seiner "politischen« oder "geistigen« Bestimmung als soziales Mitglied nicht aufgeht. Wie auch immer unklar artikuliert und unzureichend begründet, bildet dies die andere Seite der antitotalitären Erbschaft des Begriffs der Menschenwürde (dieses Motiv findet sich bei so unterschiedlichen Autoren wie Adorno, Plessner und Luhmann. ${ }^{17}$ Darin steht der "Mensch« für die »andere Seite« (Luhmann) im Subjekt, die nicht ganz sozial integriert werden kann. Die Würde des Menschen besteht in dieser zweiten Lesart in dem berechtigten Anspruch des Menschen, außerhalb der Gesellschaft zu bleiben, und die Form der subjektiven Rechte findet eben darin ihre Begründung, dass sie diesen Anspruch für alle gleichermaßen zur Geltung bringt, indem sie der nicht normativ regelbaren Willkür des Einzelnen einen Spielraum gibt.

\section{Das Paradox des Menschen und die Dialektik der Rechte}

11 Rechtslegitimatorische Positionen entscheiden sich zumeist für eine der beiden Lesarten der subjektiven Rechte und beschreiben die Rechte entweder als Ermöglichung sozialer Mitgliedschaft oder der individuellen Willkür. Dagegen betonen rechtskritische Positionen, die ihre Wurzeln im Kommunitarismus ${ }^{18}$ oder im Marxismus ${ }^{19}$ oder in beidem ${ }^{20}$ haben, die »dialektische« Verschlingung der sozial befähigenden Absicht subjektiver Rechte mit ihrer faktischen Freigabe privater Willkür. Sie tun dies mit der kritischen Absicht, die Freigabe sozialer Herrschaftsverhältnisse offen zu legen, die mit der rechtlichen Freigabe der privaten Willkür verbunden ist (wenn sie nicht gar ihr eigentliches Ziel ist): Indem subjektive Rechte die private Willkür von jeder normativen Kontrolle freisetzen, entziehen sie zugleich die sozialen Herrschaftsverhältnisse der Kritik. Diese dialektische Verknüpfung von Freiheit und Herrschaft gilt aber auch für die andere Seite der subjektive Rechte: Auch die Ermöglichung sozialer Mitgliedschaft ist immer zugleich befähigend und damit befreiend, wie sie normierend und damit disziplinierend ist. Das lässt sich als Hinweis darauf lesen, dass es gerade die dialektische Verknüpfung verschiedener Seiten ist, die die Form der subjektiven Rechte ausmacht. Darin liegt der Wert oder die Leistung dieser Form für moderne Gesellschaften: In ihr sind Freiheit und Herrschaft unlösbar miteinander verknüpft, und zwar weil sie jeweils für die Form subjektiver Rechte in beiden Lesarten zutreffen: für die subjektiven Rechte als Ermöglichung sozialer Mitgliedschaft wie als Freisetzung 
individueller Willkür. Eine Analyse des Begriffs der Menschenwürde kann dazu beitragen, den Grund der Unauflösbarkeit dieser rechtskonstitutiven Spannung zu verstehen. Dieser Grund ist die paradoxe Gestalt des Menschen als Träger der Rechte.

\section{BIBLIOGRAPHIE}

Adorno, T.W. (1997): »Aldous Huxley und die Utopie«, in: Gesammelte Schriften Bd. 10, Frankfurt a.M., Suhrkamp.

Agamben, G. (2001): »Jenseits der Menschenrechte«, in: ders.: Mittel ohne Zweck, Freiburg / Berlin, Diaphanes, S. 23-34.

Alexy, R. (1994): Theorie der Grundrechte, Frankfurt a.M., Suhrkamp.

Arendt, H. (1949): ")The Rights of Man«. What Are They?«, in: Modern Review, 3(1), S. 24-36.

Berlin, I. (1969): Four Essays on Liberty, Oxford, Oxford University Press.

Böckenförde, E.-W. / Spaemann, R. (Hg.) (1987): Menschenrechte und Menschenwürde. Historische Voraussetzungen - säkulare Gestalt - christliches Verständnis, Stuttgart, Klett-Cotta.

Brown, W. (2002): „Suffering the Paradoxes of Rights”, in: Brown, W. / Halley, J. (Hg.): Left Legalism / Left Critique, Durham / London, Duke University Press, S. 420-433.

Dürig, G. (1956): »Der Grundrechtssatz von der Menschenwürde«, in: Archiv des öffentlichen Rechts, 2, S. 117-157.

Enders, C. (1997): Die Menschenwürde in der Verfassungsordnung. Zur Dogmatik des Art. 1 GG, Tübingen, Mohr (Siebeck).

Feinberg, J. (1980): Rights, Justice, and the Bounds of Liberty, Princeton, Princeton University Press.

Glenden, M.A. (1991): Rights Talk, New York, The Free Press.

Geuss, R. (2005): Outside Ethics, Princeton, Princeton University Press.

Habermas, J. (1992): Faktizität und Geltung. Beiträge zur Diskurstheorie des Rechts und des demokratischen Rechtsstaats, Frankfurt a.M., Suhrkamp.

Habermas, J. (1999): »Zur Legitimation durch Menschenrechte«, in: Brunkhorst, H. / Niesen, P. (Hg.): Das Recht der Republik, Frankfurt a.M., Suhrkamp, S. 386-403.

Haucke, K. (2003): Das liberale Ethos der Würde, Würzburg, Königshausen \& Neumann.

Hegel, G.W.F. (1970): Grundlinien der Philosophie des Rechts, Frankfurt a.M., Suhrkamp.

Höffe, O. (2002): Medizin ohne Ethik? Frankfurt a.M., Suhrkamp.

Honneth, A. (1992): Kampf um Anerkennung. Zur moralischen Grammatik sozialer Konflikte, Frankfurt a.M., Suhrkamp.

Kelsen, H. (1960): Reine Rechtslehre, Wien.

Luhmann, N. (1965): Grundrechte als Institution, Berlin, Dunker \& Humblot. 
Luhmann, N. (1981): „Subjektive Rechte: Zum Umbau des Rechtsbewußtseins für die moderne Gesellschaft«, in: ders.: Gesellschaftsstruktur und Semantik, Bd. 2, Frankfurt a.M., Suhrkamp, S. 45104.

Luhmann, N. (2005): »Die Tücke des Subjekts und die Frage nach dem Menschen«, in: ders.: Soziologische Aufklärung, Bd. 6, Wiesbaden, VS, S. 149-161.

MacIntyre, A. (1981): After Virtue, Notre Dame, University of Notre Dame Press.

Marshall, T.H. (1964): Class, Citizenship, and Social Development, Garden City/ N.Y., Doubleday.

Menke, C. / Pollmann, A. (2007): Philosophie der Menschenrechte. Zur Einführung, Hamburg, Junius. Sen, A. (1992): Inequality Reexamined, New York / Oxford, Russell Sage Foundation u.a.

Weber, M. (1980): Wirtschaft und Gesellschaft. Grundriß der verstehenden Soziologie, hg. v. Johannes Winkelmann, Tübingen, Mohr (Siebeck).

Wellmer, A. (1993): »Freiheitsmodelle in der modernen Welt«, in: ders.: Endspiele: Die unversöhnliche Moderne. Frankfurt a.M., Suhrkamp, S. 15-53.

Wetz, F.J. (2005): Illusion Menschenwürde. Aufstieg und Fall eines Grundwerts, Stuttgart, Klett-Cotta. Zimmermann, R. (2005): Philosophie nach Auschwitz. Eine Neubestimmung von Moral in Politik und Gesellschaft, Reinbek bei Hamburg, Rowohlt.

\section{NOTES}

1. Weber (1980), S. 398; vgl. Kelsen (1960), Kap. 29.

2. Weber (1980), S. 419.

3. Alexy (1994), S. $211 \mathrm{ff}$.

4. Habermas (1992), S. $142 \mathrm{f}$.

5. Sen (1992); vgl. Honneth (1992), Kap. 5, im Anschluss an Marshall (1964).

6. Berlin (1969); vgl. Geuss (2005).

7. Feinberg (1980), S. 156-158.

8. Luhmann (1981), S. 66.

9. Vgl. Wellmer (1993).

10. So etwa Habermas (1999).

11. Menke / Pollmann (2005); Wetz (2005); Zimmermann (2005).

12. Vgl. Böckenförde / Spaemann (1987); Dürig (1956).

13. Vgl. Höffe (2002).

14. Hegel (1970), § 57.

15. Arendt (1949); vgl. Agamben (2001).

16. Vgl. Enders (1997).

17. Adorno (1997); zu Plessner vgl. Haucke (2003); Luhmann (2005).

18. Glenden (1991).

19. Brown (2002).

20. MacIntyre (1981). 
INDEX

Mots-clés : droits de l'homme, droits (naturels), droits (subjectifs), dignité humaine

Schlüsselwörter : Menschenrechte, Rechte (natürliche), Rechte (subjektive), Menschenwürde

\section{AUTEUR}

\section{CHRISTOPH MENKE}

Christoph Menke ist Professor für Philosophie an der Universität Potsdam. Nähere Informationen finden Sie hier. 